Gewalt im Jugendgefängnis: „Siegburg ist keine Justizpanne, das ist eine Strafvollzugskatastrophe"

\title{
Verflucht sind die Fische
}

Wer Schwäche zeigt, wird Opfer und ist den Ritualen der Verrohung ausgeliefert - wie trotzdem versucht wird, mit weniger Druck mehr zu erreichen

\begin{abstract}
$\Lambda$ delsheim, im Dezember 2006 - Der Tag, an dem Hermann Heibach lernte, was es heißt, im Gefängnis der Schwächere zu sein, war ein Samstag. Es war ein verhangener Tag, trostlos wie Wochenenden sind in der Justizvollzugsanstalt Siegburg, keine betreute Freizeit, keine Besucher, kein Sport. Ein Tag ohne Ende: vier Insassen auf 20 Quadratmetern, 23 Stunden lang. Ätzende Langeweile. Unterbrochen nur von einer Stunde Hofgang, weil es das Gesetz so vorschreibt. Das Abendbrot bekam Hermann Heibach in einer Plastiktüte mit dem Mittagessen. Es war 13 Uhr, es war der 11. November, vier Tage davor waren zwei Jungs aus Bottrop neu in seine Viererzelle gekommen.
\end{abstract}

So fingen sie an, die letzten Stunden im Leben von Hermann Heibach.

Sechs Monate sollte er im Gefängnis bleiben. Ein Kleinkrimineller, in Heimen aufgewachsen. Ein Gewächs aus dem Prekariat. Er klaute Bier, Essen, Motorroller. Irgendwann überfiel er mit einem Kumpel einen Kiosk in Leverkusen, für drei Flaschen Schnaps und Zigaretten. Er drohte mit dem Messer, wurde gefasst, zu 80 Sozialstunden im Krankenhaus verurteilt, am vierten Tag hatte er keine Lust mehr. Haftbefehl. Im Oktober fand ihn die Polizei am Kölner Hauptbahnhof. Er kam ins Gefängnis Köln-Ossendorf, dann nach Siegburg. Freunde sagen, er war ein Tolpatsch, der den Mund manchmal zu voll nahm.
Sechs Monate, es schien eine überschaubare Zeit zu sein.

Aber am 11. November geschah, was Experten den Hunger nach einem perversen Kick nennen. Vier frustrierte, gelangweilte, zornige junge Männer, die Rankings aufstellen und Koalitionen schmieden, zwei gegen zwei, zusammen gegen das System - oder alle gegen einen. Irgendwann beschlossen drei von ihnen, den Schwächsten zu töten, einfach so, wie man beschließt, ins Kino zu gehen.

Es war Samstag, es gab nicht viel anderes zu tun.

\section{So viele Großmäuler}

„Der war halt 'n Fisch”, sagt einer in der Kantine der Justizvollzugsanstalt Adelsheim, drei Autostunden weiter südlich. Adelsheim ist das zweitgrößte Jugendgefängnis Deutschlands, 445 Insassen, auch ein Knast, auch belegt mit frustrierten, gelangweilten, zornigen jungen Männern. Sie wissen von dem Mord in Siegburg. Krasser Fall, sagen sie. Wäre hier nicht möglich, sagen sie. Die wollten einen auf Trauma machen, sagen sie. Es gibt Gerüchte, dass man einen in der Zelle hängen sehen muss, dann kommt man früher raus. Es gibt so viele Gerüchte, so viel Knastgeschwätz, so viele Großmäuler, sie haben gelernt, dass man sich damit schützen kann. Von Angst redet hier keiner.

Denn Angst haben heißt Fisch sein. So nennen sie im Knast die Opfer, die abgezogen werden, die jeden Einkauf abgeben
Karin Steinberger

sakern während der Befreiungskriege in Eritrea, der Junge stutzt. Kein Eritreer wurde massakriert, man sei ein Siegervolk. „Da müssen sie falsch informiert sein." Keine Verlierer.

\section{Raushalten, stillhalten}

Es gibt die Regeln der Anstalt und es gibt die Regeln der Insassen. Irgendwo dazwischen muss man überleben. Das weiß jeder. Raushalten, stillhalten. Wer das nicht macht, ist entweder dumm oder neu. Neuen geht es nicht gut, in keinem Knast.

Joachim Walter sitzt in seinem Büro gleich über dem gigantischen Gefängniseingangstor. Er hat einen guten Blick von hier oben auf seine Anstalt, die JVA Adelsheim, Grünflächen, Häuser, dahinter die Betonmauer, 1,3 Kilometer, ohne Natostacheldraht obendrauf. Alle acht Jahre schafft es einer über die Mauer. Walter nimmt das in Kauf. Er macht hier einiges anders als andere Gefängnisleiter. Aber Knast ohne Gewalt, da muss man schon sehr naiv sein, um daran zu glauben. Andererseits, so ein Fall wie in Siegburg, das ist ein Extremfall. Den Tötungswillen, den gibt es normalerweise nicht.

Im Hintergrund räumen die Jungs ihre Blechtabletts ab, es gab Schweinswurst, zwei Scheiben Brot, Sauerkraut. Die Russen saßen wie immer rechts hinten, die Türken vorne, die gemischte Gruppe links. Fische erkennt man daran, dass sie die Tabletts für andere abtragen. Man kann sie sehen, sprechen nicht. Wer drinnen zugibt, Opfer zu sein, ist verloren. Solange es irgendwie erträglich ist, sagen sie nichts.

„Es gibt hier kein Problem”, sagt ein Junge, er kommt aus Eritrea, ist 20 Jahre alt, seit vier Monaten in Adelsheim, ein Winzling. Er redet viel: „Du musst dich nur gescheit anpassen, musst höflich reden und nicht lästern. Und verpfeifen, das macht man nicht." Sagt er und wischt den Boden. Ein Beamter fragt ihn nach den Mas-
Walter sagt: „Es ist wie beim Autofahren. Wenn man ein Schild aufstellt, auf dem steht, Achtung Radarkontrolle, will man, dass die Leute langsamer fahren. Wenn man blitzt, will man abkassieren."

Joachim Walter will nicht Abkassieren. Für ihn sind seine Insassen keine Defizitwesen, keine Sozialbehinderten, auch keine kleinen Erwachsenen. Es sind Jugendliche, die Mist gemacht haben. Zug statt Druck, das ist seine Philosophie. Jeder Gefängnisdirektor hat eine eigene Philosophie. Die von Walter heißt: Den Esel nicht von hinten schlagen, sondern vorne mit Karotten locken.

„Ich halte nichts davon, alle hinter Gitter, Stahltüre zu. Bei 
uns finden sie keine Bereichsabtrennungen, viele Zellen sind ohne Gitter. Wir haben trotzdem alle im Auge", sagt Walter. Es gilt, die Geschlossenheit zu relativieren. In Adelsheim gibt es fast nur Einzelzellen. Alle mit Blick über die Mauer, hinaus ins Land. Das war schon mal anders. Als Adelsheim überbelegt war, gab es auch hier Dreierzellen und Scheinhinrichtungen. Platzmangel ist das Schlimmste, da nützt die beste Philosophie nichts, die beste Zugangskommission, da kann man keine Gruppe mehr komponieren, da kommt halt Ladendieb zu Mörder, Frischfleisch zu Profi. Da kann man nur hoffen. Das Zweitschlimmste ist, wenn nicht genug Beamte da sind, fähige Beamte, die mitbekommen, wenn sich einer nicht mehr hinsetzen kann, weil er vergewaltigt wurde; die erkennen, ob blaue Flecken von einem Sturz kommen oder von Schlägen; die spüren, wenn einer Hilfe braucht.

Das hat mit Geld $\mathrm{zu}$ tun und mit baulichen Möglichkeiten. Aber auch mit der Einstellung des Anstaltsleiters. Und mit den Karotten, die er anbietet.

Joachim Walter setzt auf Belohnung - und auf Bildung. Durchschnittlich bleiben die Insassen 11 Monate - mindestens ein halbes Jahr, höchstens zehn Jahre. Genug Zeit, um Dinge anzustoßen. Wer in Adelsheim einen Fernseher haben will, muss die Gebühr erarbeiten. Er kann Tischler werden oder Fleischer, Maurer oder Elektriker, er kann einen Schulabschluss nachholen, den er draußen nie geschafft hat. Er kann sich hocharbeiten, vom geschlossenen Vollzug in den offenen. Walter nennt das hochdifferenzierten Strafvollzug. Jedes Haus in seinem Gefängnis hat einen Buchstaben und einen eigenen Charakter. In E1 sind die Jüngsten, in F die Langstrafler. „Mit denen ist es am einfachsten, die müssen sich auf uns einlassen", sagt Walter.
Wer keinen Schlaf findet, wird die Nächte hassen. Die brandgehemmten, dünnen Schaumstoffmatratzen, die ausgeleierten Schlafanzüge, das aufdringliche Licht der Scheinwerfer, das Geschrei der Untersuchungshäftlinge, die nachts an ihren Gitterstäben hängen wie Spinnen und irgendwas hinausschreien in die Dunkelheit. Namen, Fernsehprogramme, prollige Sprüche, wenn eine Beamtin vorbeikommt. Alles krakeelen sie hinaus in die Nacht von Adelsheim, nur, um nicht allein zu sein mit sich und der Zeit.

Walter weiß, dass Zeit Strafe ist. Deswegen gibt es neben der Arbeit noch andere Dinge. Es gibt eine Rap-Gruppe, eine Gefängniszeitung und Blumensteckgruppen bei Hilde Kilian, die sie „Knastomi” nennen. Bei ihr hocken zwischen Glitzerengelchen Mörder und Vergewaltiger. „Sie glauben nicht, wie schön es ist, im Knast zu arbeiten, was sie hier an Kraft kriegen", sagt Hilde Kilian. Die zwei Jungs bei ihr kommen aus Haus F. F ist wie eine Krankheit. Wer dort ist, ist Mörder oder Vergewaltiger, Held oder Abschaum.

Die aus F gehen nicht oft raus. Sie haben ihr eigenes Haus, ihren eigenen Hofgang. Und sie haben Sozialtherapie, das kann im Gefängnis auch schwer kränkend sein und hoch bedrohlich. Männlichkeitsideale zu verhandeln, gehört sich in der Gruppe nicht. Aber die Jungs sind cool, sie sagen, die anderen Häuser, das sei „Legoland”, zu laut, zu nervig, Kindergarten. Was sie nicht sagen, dass Legoland gefährlich ist, Vergewaltiger sind da Freiwild. Nur zu Hilde Kilian gehen sie, Gestecke machen.

Und dann gibt es noch G3. Dicht an der Mauer steht es, das Just Community Projekt, ein Holzhaus. Basisdemokratie mitten im Knast. 15 Insassen, ein paar Beamte, jeder Mann eine Stimme. Es gibt einmal in der Woche Vollversammlung, es gibt ein Leitungskomitee, ein
Fairnesskomitee. Wenn das Klo dreckig ist oder der Müll falsch sortiert, gibt es einen Beschluss, oft erstaunlich streng. Man probiert, diskutiert, man schafft das gemeinsame Frühstück $a b$, dann führt man es wieder ein. Wer drei Minuten zu spät kommt, hat einen Tag Küchendienst. Einmal im Monat darf man raus, einkaufen.

G3 ist Gefängniselite. Die Elite hockt herum, schaut fern, stöhnt. Ständig drohe im Hintergrund der geschlossene Vollzug. Ein Joint und man ist weg. „Hier wirst du als Mensch gesehen, aber es ist anstrengend." Zustimmendes Murren. "Aber wir sind die Vorbilder im Knast." Stolzes Murren. Das Modell beruht auf Kommunikation, auf Eigenverantwortung. Im Gefängnis bekommt man Struktur normalerweise vorgesetzt, in G3 befolgen sie ihre eigenen Regeln. Gewalt gibt es selten, sie strengen sich an. Walter sagt: „Dass es erfolgreich ist, ist unbestritten. Aber in Zeiten, in denen man Wahlen gewinnt, indem man härtere Strafen propagiert, ist das schwierig."

Die Gesellschaft will, dass das Gefängnis schmerzt. Walter will, dass es hilft.

Er kennt die Entwürfe für das neue Jugendstrafvollzugsgesetz, auch den bayerischen, der den Schutz der Allgemeinheit das erste Mal vor das vom Bundesverfassungsgericht geforderte Ziel setzt, Inhaftierten ein künftiges straffreies Leben in Freiheit zu ermöglichen. Es klingt wie eine Kleinigkeit, aber es verschiebt die Prioritäten. Das macht ihm Sorgen.

Walter holt eine Flasche aus dem Regal. Unten ist sie dunkelblau, oben heller, darauf eine Plastikhaube. „Das ist ein geschlossenes System, wie Adelsheim", sagt er. Die Flasche ist voller Wasser, eine Glasfigur schwimmt darin, der Cartesische Taucher, er sieht aus wie ein Teufel. Wenn Walter nichts macht, hängt die Figur oben im Flaschenhals, als wollte sie raus. Drückt Walter mit dem Daumen auf die Haube, taucht sie ab. „So ist das, wenn man Druck ausübt", sagt er, presst weiter, bis der Teufel ganz unten aufschlägt und hinter dunkelblauen Wänden verschwindet. „So ist das auch im Gefängnis, je mehr Druck wir ausüben, desto weiter tauchen die Jungs ab, in die Subkultur." Dorthin, wo sie keiner mehr sehen kann. Dorthin, wo sie auch keiner mehr schützen kann.

Auch Hermann Heibach konnte keiner mehr schützen. Es war Samstag, es waren vier Vollzugsbeamte für 715 Gefangene in der JVA Siegburg. Es war das erste Wochenende mit den zwei Neuen in der Zelle. Es war wie immer, wenn sich im Gefängnis die Konstellationen verändern, es war ein Kampf um Vorherrschaft, eine Festlegung der Hackordnung.

Es sah von Anfang an nicht gut aus für einen wie Hermann Heibach.

Es gibt so viele Knastrituale: Verräter sind „Einunddreißiger”, Kinderficker „Sittiche”, es gibt Fensterrambos, die aus den Zellen heraus Sprüche machen, Opfer werden zum Weib gemacht. Und dann gibt es die Neuen, Frischfleisch. Alle wittern frisches Fleisch. Und alle hassen Vergewaltiger. Beim Hofgang gibt es Spiele, die Türken im Kreis, die Russen im Kreis, wenn ein Falscher durchgeht, ist er dran. Wer diesen Machtspielen ausweicht, ist Opfer. Aber Opfer sein, ist absolutes Tabu. Draußen sind die Spielregeln anders, da kann man gehen, hier nicht, hier ist täglich Showdown. Deutsche heißen „Kartoffeln”. Die russischen Aussiedler „Russenblock". Sie haben Tattoos an den Fingern und strenge Regeln, sie halten zusammen, versorgen sich gegenseitig. Sie kennen Schlimmeres. Wer einem Beamten die Hand gibt, ist bei den Russen ein Verräter.

Hermann Heibach war ein hagerer Bursche, ein bisschen tap- 
sig, keiner, der zuschlägt. Pech gehabt.

Der Tag, an dem er lernte, dass man sich nach dem Tod sehnen kann, war ein Samstag. Zu dritt schlugen sie ihn, sie flößten ihm Urin ein, zwangen ihn, eine Tube Zahnpasta $\mathrm{zu}$ essen und aus dem Toilettenbürstenhalter Urin und Spucke zu trinken. Sie ließen ihn sein Erbrochenes vom Boden auflecken, ließen ihn Abschiedsbriefe schreiben und machten aus Kabeln Galgenstricke, an denen er sich aufhängen sollte. Zwei davon rissen.

In Siegburg, sagt ein Junge, der drin war, herrsche das Faustrecht, eine Art innere Anarchie, die Beamten seien unmotiviert, oft krank, der Umgangston brutal. Siegburg, heißt es, ist die härteste Anstalt, vom Klima her. Zellen in langen Gängen, laut, königlich-preußisch, eine Architektur der Einschüchterung. Irgendwann gelang es Hermann Heibach, den Notruf in der Zelle zu drücken. Aber was heißt das schon, am Wochenende schlagen die Jungs ständig aus Langeweile Alarm, meist kommt nichts aus der Gegensprechanlage als heiße Luft. Ein Beamter meldete sich, fragte, die Täter sagten: Fehlalarm.

Das war das Todesurteil. Fehlalarm.

Klaus Jünschke sitzt in einer kleinen Dachgeschosswohnung in Köln. Er kennt diese Knöpfe, die falsche Sicherheit suggerieren, er kennt die Beamten, die oft wegsehen, die brutalen Mithäftlinge, Schatten von Menschlichkeit. Er kennt die Geräusche der Gefängnisschlüssel vor dem Hofgang, die Blicke der Beamten bei der Lebendkontrolle am Morgen, die Enttäuschung über Freunde draußen, die einen fallenlassen, die Hitzeschübe, wenn Besuch angekündigt ist. Zwei Meter mal vier Meter. Bett, Tisch, Waschbecken. Jeden Tag: Bett, Tisch, Waschbecken. Was hat er seine Zellenwand angestarrt, von Vorgängern bearbeitet.
Jünschke kennt den Unterschied zwischen Stahltüren und Holztüren, bei denen man sich bei Panikattacken zumindest ausmalen kann, wie man sie eintritt. Er weiß, was es heißt, wenn sie vor die Normalvergitterung am Fenster noch Feinvergitterung hängen. Vermeintliche Kleinigkeiten, lebensbestimmend, weil man nicht mal mehr mit Schnüren von Zelle zu Zelle „Pendeln” kann, Feuerzeug oder Tabak oder Zettel.

„Wir sind hier im Thema”, sagt Klaus Jünschke, kein Lächeln.

\section{Nur kein Knastkitsch}

16 Jahre war er im Gefängnis. Auf den Fahndungsfotos der Baader-Meinhof-Gruppe trägt er einen Schnauzer und einen Pullover mit zwei weißen Streifen am Kragen. Am 22. Dezember 1971 überfiel er mit sechs anderen RAF-Mitgliedern eine Bank, der Polizist Herbert Schoner starb. Am 9. Juli 1972 wurde er festgenommen. 1988 begnadigt. 16 Jahre.

Jünschke ist schon lange im Thema.

Sein Codename war „Spätlese”. Er hat ihn von Gudrun Ensslin bekommen. Ihre Schwester Christiane sitzt mit ihm im Dachgeschoss, sie arbeiten zusammen an einem Buch über jugendliche Straftäter und an einer Ausstellung über den Zellenalltag. Jünschke ist für die Grünen Mitglied im Gefängnisbeirat, einer Art Mittler zwischen drinnen und draußen. Er kümmert sich um junge Menschen in Haft. Die ganze Wohnung ist eine Bibliothek des Immergleichen: „Gespräche mit Eingeschlossenen” - „Frauen im Gefängnis” - „Innenansichten aus dem Knast". Unter dem Schreibtisch hat er einen Ordner der Skandale: 1967 sterben zwei Häftlinge im Kölner Klingelpütz, 2005 zünden zwei Zellengenossen einen Mithäftling in der JVA Recklinghausen an, 2006 reißen Zellengenossen einem Mithäfling die Fußnägel aus in der JVA Oslebshausen. Es gibt viele gruselige Geschichten. „Wir sind dafür, dass Jugendliche nicht eingesperrt werden", sagt Klaus Jünschke.

Noch etwas sagt er, es gehe hier nicht um Knastkitsch, keiner behaupte, dass da nur Unschuldige sitzen. Aber man müsse schon auch fragen, wie diese Menschen zugerichtet wurden, um solche Brutalos zu werden. Von seelischer Hygiene redet er, von Menschen statt Mauern, von falschen Freunden, die man da drinnen zwangsläufig kennenlerne, von immer weniger Eltern, die ihre Kinder im Gefängnis besuchen und davon, dass man auch sehen müsse, was dort alles nicht passiere. Jünschke hat viel geschrieben seit Siegburg, er hat angeprangert, dass es keine jugendorientierte Pädagogik hinter den Mauern gibt und dass es nicht reiche, sie wie Zootiere zu verwahren, man müsse sie schon auch beschäftigen. Er hat den verstorbenen Anstaltsleiter Jörn Foegen zitiert, der immer betont hat, dass er Gefängnisdirektor ist, nicht Klinikchef, und dass Süchtige nicht in den Knast gehörten. „Es gibt Polizeireporter, Gerichtsreporter, aber Gefängnisreporter gibt es nicht. Keiner fragt, was geschieht nach dem Urteil? Deckel zu, Affe tot", sagt Jünschke.

Dann muss er weiterarbeiten, das Buch ist ihm wichtig. Zu seiner Geschichte sagt er nur so viel: „Das Schlimmste ist die Ohnmacht. Die Vorstellung, wie in New Orleans das Wasser kommt und die Beamten weglaufen. Man muss es vergessen, sonst wird man verrückt. Diese Schmerzen kann man nicht ausdrücken."

\section{Berauscht vom Entsetzen}

7100 Insassen sind in Deutschland im geschlossenen Jugendstrafvollzug. Davon 10 bis 15 Prozent unter 18 Jahre alt, die Hälfte ist 18 bis 20 Jahre alt, der Rest 21 bis 24 . Christian Pfeiffer vom Kriminologischen For- schungsinstitut Niedersachsen sagt: „Siegburg ist keine Justizpanne. Das ist eine Strafvollzugskatastrophe." So viele Fehler wurden gemacht. Drinnen. Und so viele werden gemacht. Draußen. Pfeiffer sagt, die Dinge werden eigentlich immer besser, aber die Leute wissen es nicht. Die Zahlen an Straftaten gehen runter, und trotzdem gebe es 40 Prozent mehr Gefangene als vor zehn Jahren. „Das ist völlig sinnlos. Es ist eine politisch gewollte Überfüllung", sagt Pfeiffer.

Siegburg ist für ihn eine Botschaft an alle, nicht nur an die Gefängnisse.

Normalerweise gibt es Abschiedsschläge, jeder bekommt sie. Es ist das Endritual, die letzte Bedrohung, wenn die anderen sich aufstellen, links und rechts, wenn sie schlagen, fest und wahllos. Für Hermann Heibach stellte sich keiner mehr auf, er verließ Siegburg im schlichten Kiefernsarg.

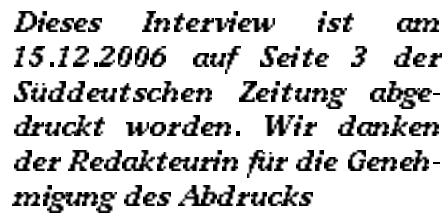

